\title{
A Novel Path Planning System for Guidance of Blind People using Integration of GA and $A^{*}$ Techniques
}

\author{
Mohamed M. Ghoniem \\ Computer Science Department \\ Mansoura University, Egypt
}

\author{
A. E. El-Alfi \\ Computer Science Department \\ Mansoura University, Egypt
}

\author{
A. F. Elgamal \\ Computer Science Department \\ Mansoura University, Egypt
}

\begin{abstract}
It is a challenging task for the blind people to perceive indoor environment information and walk independently. Path planning is a crucial requirement to provide constant assistance for blind people to navigate from one location to another. This paper presents a novel system for path planning to assist blind people for walking through the indoor environment and avoiding the obstacles. The algorithm is based on genetic algorithm and $\mathrm{A}^{*}$ algorithm to select the shortest path that achieves the lowest computational time. A comparison a mong the GA, A* and the new algorithm shows that is faster and shorter than the previous algorithms.
\end{abstract}

\section{Keywords}

Path planning, grid map, Genetic Algorithm, A Star algorithm, Path guidance.

\section{INTRODUCTION}

Artificial Intelligence and path planning have fully assisted in the utilization of several robotic applications like vacuum cleaner [1], underwater [2], aerial vehicles [3] and blind people [4]. In motion planning, the principle issue is to compute the path which allows movement from any starting point to reach a goal point within the place while avoiding obstacles.

This issue is addressed in previously published studies as path planning problem $[5,6]$, and requires high computational complexity because it is a Non-deterministic Polynomial. In real-life, determining feasible solutions in the critical applications like autonomous mobile robots [7] supposes to solve path planning problems effectively.

In daily life, humans need to acquire information from surrounding environment. The majority of information is acquired by eyes.

However, the World Health Organization (WHO) has announced that there are 285 million people who are visually impaired all over the world. Among them, 36 million of the blind, and 246 million suffering from low vision. Moreover, $82 \%$ of the blind people are aged 50 and above [8]. Loss of vision is a critical issue that prevents blind persons to live independently and raises the unintentional injuries risk which often causes medical consequences [4]. Besides that, only with a physical support could blind persons walk safely. Therefore, the need to assist the visually impaired persons both to be aware of environment information and to freely walk through navigation message is crucial [9].

Taking the vacuum cleaner robot as a path planning example, which sweeps each accessible area within the indoor environment, this mechanism is referred as a coverage region path planning [10]. For more path planning examples, there are increasing trends towards developing Autonomous Underwater Vehicles to reduce the costs of ocean survey and also minimize the risks of sending humans to the hostile environment in the deep seas with unpredictable weather state and the higher pressure at greater depth [11]. In addition, path planning is used also for Unmanned Aerial Vehicles (UAVs), which indicates that according to the UAV maneuver performance, hit probability, penetration probability, flight timing within bounds, it can help to search for a feasible or optimal path from any initial/starting point to the final/target point flight [12].

The path planning methods shown in the previous literature are divided into two major approaches: classical approach as well as heuristic approach (utilizing Artificial Intelligence Technique) [13]. Prominent classical planning approaches comprise the Potential Field Method [14], Probabilistic Roadmap (PRM) [15], Grid Based Method [16], Rapidly Exploring Random Tree [17] and. heuristic planning approaches involve fuzzy logic [18], artificial neural network [19], ant colony algorithm [20], genetic algorithm (GA) optimization [21] and the approaches based on particle swarm optimization [22]. Each approach has its own drawbacks and so far, there is no particular method that can fully solve path planning issue. Thus, studies investigate recently new more efficient and robust solutions or seeking to enhance existing methods.

Therefore, this paper introduces a proposed path planning algorithm based on GA including $\mathrm{A}^{*}$ as fitness function to select the shortest optimal path that achieves the lowest computational time. The rest of the paper is organized as: Section 2 shows an overview of some published works addressing the existing path planning algorithms. The proposed algorithm is illustrated in Section 3. Section 4 shows the discussion on experimental results. Finally the conclusion and references are presented.

\section{RELATED WORK}

The path planning is a crucial component of navigation systems that can influence their overall performances. The path requires to be planned in such a way that maximizes the usability and success ratio while reducing the opportunity of the users getting lost. The smartest path planning method requires regard the user's requirements, then customizing the path accordingly [23].

Recently, several works have been presented to solve the path planning problems appear in widely fields. An overview about the previously published works on path planning systems in general, and those presented for assisting the blind people are presented in Table 1. 
Table 1. Literature review on Path planning algorithms and systems

\section{Algorithm}

A*

Dijkstra's

Modified Dijkstra's

D* Lite

Theta*

GA

PSO

PSO-LPM

RRT

DAALO

PRM

\section{Area}

Blind people

Blind people

Blind people

Autonomous Underwater Vehicles

Blind people

vacuum cleaner robot

vacuum cleaner robot

Unmanned Aerial Vehicle

Mobile Robot

Blind people

Mobile Robot

Autonomous Underwater Vehicles

Autonomous Underwater Vehicles

Unmanned Aerial Vehicle

Autonomous Underwater Vehicles

Unmanned Aerial Vehicle

Mobile Robot

Unmanned Aerial Vehicle

\section{Problem solution}

An indoor wheelchair navigation system.

A navigation device to assist the visually impaired groups.

An integrated human-computer interaction system which assists the visually impaired to navigate.

Path planning algorithm to help the visually impaired persons.

An approach to solve motion planning within a dynamic environment.

Navigation for the visually impaired system based on wearable RGBD.

Online complete coverage approach developed for autonomous cleaning robot.

A coverage region path planning for the vacuum cleaner robots.

An approach to plan the quadrotor path.

Reference

A solution to path planning problem and environment modeling.

An optimized path planning method for solve path planning problem.

A path planning approach with predetermined waypoints for blind people.

A path planning approach for solving the issue of real-time path planning.

A method for solving the route planning issue.

A solution for routing planning problem.

A path planner using a hybrid optimization algorithm.

An innovative algorithm for computing the optimum risk path of unmanned aerial vehicle.

A routing optimization method for path planning.

An approach for solving the route planning problem.

A path planning approach to enhance mobile robot navigation abilities.

A method to implement low-cost path planning for the Unmanned Aerial Vehicles. 
From the previous studies, the conventional algorithms employed for path planning problem have major drawbacks i.e. higher computational complexity. The majority of the related works, in particular, concentrates the problems of task and target assignment as well as time scheduling and do not consider the requirements of safe path.

In the previously discussed research,

1 It is clearly seen that A*, Dijkstra's, Theta* and D* Lite algorithms are the best algorithms regarding the shortest distance with fewer edges. Nevertheless, these algorithms require a long computational time in order to obtain the shortest distance.

2 RRT algorithm increases the number of edges in the path.

3 PRM and PSO cannot completely cover the entire free space and the random distribution.

4 GA produces smoother navigations but requires a long computational time.

Among these approaches GA and $\mathrm{A}^{*}$ are the most popular in finding optimal solutions for difficult combinatorial problems.

\section{BACKGROUND}

\subsection{Genetic Algorithms for path planning}

GAs were originally evolved in 1960 by John Holland [3]. They are one of the natural inspired algorithms [42-44]. GAs are based upon the Darwinian evolution concepts which include an initialization stage, fitness function for chromosome evaluation, natural selection method, crossover process, and mutation operator [45]. The main steps of the standard GA for path planning are executed as follows [46]:

- An appropriate genetic representation of individuals (chromosomes).

- A method for generating an initial population.

- A fitness function for evaluation of the potential solution quality.

- Genetic operators which alter the parents' genetic composition to generate a new offspring.

- Determination of various GA parameter values (population size, rate of cross over, rate of mutation, stopping criteria... etc.).

- If the optimal solution is found, then end genetic algorithm and return the optimal path.

\section{2 $\mathrm{A} *$ algorithm for path planning}

A* algorithm was introduced by Hart et al. (1968) that is considered an extension to Dijkstra's algorithm [47]. A* algorithm represent one of the optimal experimented path planning algorithm that can be implemented to topological or metric configuration space [48]. The algorithm comprises the heuristic searching and shortest path-based searching. A* algorithm is indicated as best-first algorithm, as the evaluation of each cell in the configuration space is implemented by [49]:

$f(v)=h(v)+g(v)$

Where $h(v)$ represents the heuristic distance (Chebyshev Euclidean or Manhattan) from the cell to the target state and $g(v)$ denotes the path length from the initial point to the goal point through the selected cells sequence.

Supposing the start point is $\mathrm{S}$ and the end point is $\mathrm{T}$, the searching procedure of $\mathrm{A}^{*}$ algorithm is expressed as [50]:

1. Start from the initial point $S$. Let it represent the current point that will be treated and join it to the open list.

2. Search for all reachable points between the current point neighborhoods ( 8 neighbors). Add them to the open list then let their parent point be the current point.

3. Delete current point from open list then join it to the closed list, which stores all points that do not require to be searched anymore.

4. Find the optimum point $P$ in the open list using the evaluation function $F(P)$ defined by Eq. 1 . If $P$ does not represent the target point, delete it from the open list and update the closed list with it. Then go to Step 1. Contrarily, if $P$ represents the target point, then go to Step 5.

5. Stop the searching process. Get the solved path by returning the sequence kept in the open list is.

However, there are some constraints on using GA and A* which include:

- Increasing the number of turns in the path.

- Consuming longest computation time.

- The fitness function selects only the points leading to the correct path regardless of the distance between starting and ending points.

To overcome these constraints, a path planning algorithm is proposed in this paper to select the optimal path that achieves the lowest computational time and considering the safety on walking for blind people. This algorithm is based on GA that includes $\mathrm{A}^{*}$ as fitness function.

\section{PROPOSED PATH PLANNING ALGORITHM}

The proposed system aims to decide the optimal path in an indoor environment for the motion of blind person from start point to destination avoiding the obstacles through the environment. If any obstacle is found during the navigation throughout the suggested path, the proposed system redirects the blind person to his destination throughout new optimal path.

To achieve that; first, the indoor environment representation must be well defined, second mathematical problem formulation is required and finally proposed solution technique is chosen. This techniques is based on GA and $\mathrm{A}^{*}$ as a fitness function.

\subsection{Indoor environment representation}

During the path planning procedure, the proposed system interacts with the environment for making decisions. Therefore, it requires a model to represent the environment. Multiple representations of the indoor environment have been introduced in the literature [51] like the polygon, the occupancy grid, the representation of cell tree. Through the grid-based representation [52] using a grid that is orderly numbered. In [53], the grid map has been employed, where the environment has been segmented into cells with the same size.

In this paper, a 3D map made with the measurement points acquired from a stereo camera is generated. Projecting the $3 \mathrm{D}$ 
map to the 2D grid map leads to indoor map [54]. A binary matrix is employed to model the environment into a $2 \mathrm{D}$ grid map. The model lets necessary savings in the processing and memory using. The orthogonal function $f\left(x_{i}, y_{i}\right)$ is implemented to this matrix. Each cell of the matrix is either occupied or empty. The zeroes denote empty cells from the grid map whereas as the ones denote obstacle cells in grid map, whether the cell is completely or partially occupied [55]. In figure 1 , the circles shown on the grid map represent all possible places which the blind person can occupy on the grid map.

Each cell of the map owns a coordinate which is presented by $(\mathrm{x}, \mathrm{y})$. Furthermore, the coordinate for each row in the map belongs to the domain of $x \in[0, w-1]$, where the coordinate of every column in the map belongs to the domain of $y \in$ $[0, m-1]$, where $w$ is the rows number and $m$ represents the columns number. The cell length is regarded as one unit $(u=1)$ and a cell size is sufficient for the blind person to turn around instead of moving outside it [56]. Transformation of the binary matrix into a 2D grid map is depicted in Figure 1.

After obtaining the grid map from a binary matrix, creation of a graph using the grid map is implemented. This is referred as "one hop" as one part in the path among the origin and goal nodes. Accordingly, one hop is being between each two free cell from grid map where the blind person can move in between them. Figure 2 presents a graph with 6 vertexes and 8 edges which is created from the grid map. Each single-vertex represents a free cell in the grid map which is identified by its particular coordinates. Eight neighboring points are taken for each cell. This means that blind can mov to eight directions as shown in Figure 3.

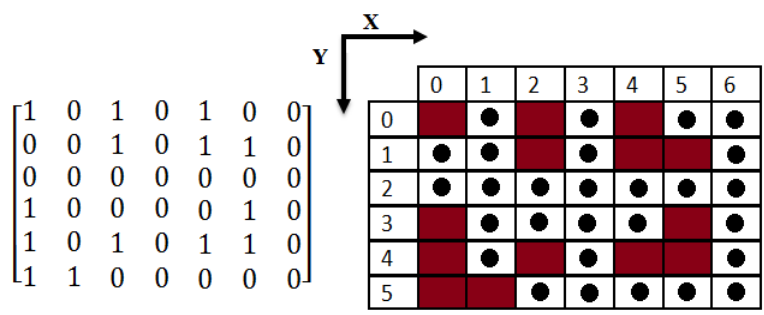

Figure 1 The binary matrix of the indoor environment.

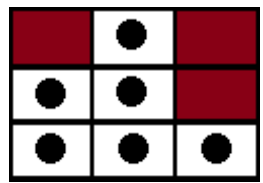

Figure 2 A grid map section
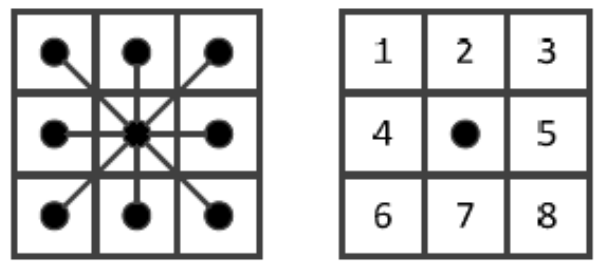

Figure 3 The 8 neighboring points of the center cell with their numbers

Accordingly, the indoor environment is modeled by 2D matrix, where every position $\mathrm{P}(\mathrm{x}, \mathrm{y})$ within the grid owns two probable values: 1 for the occupied ones and 0 for free cells. The appropriate solution represents a path (the positions set) from the initial point to the target point crossing a group of free positions that called chromosomes or population.

\subsection{Mathematical formulate of the problem}

The path starts at the initial point $(S)$ and terminates at the target point $(T)$. The path comprises the successive segments. By summing all the path segments, the entire path length is obtained. Suppose that path $P=\left[S, P_{1}, P_{2}, \ldots, P_{n}, T\right]$ comprises an initial point $\mathrm{S}$, a goal point $\mathrm{G}$, as well as $n$ consecutive points. Therefore, the path segment is produced by two consecutive points of the path. Figure 4 depicts the path.

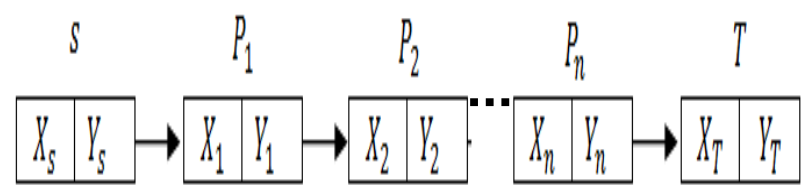

Figure 4 Total path

In order to find the path between the $S$ and $T$, a grid map is determined, it is characterized by " 0 " for free places and " 1 " for obstacles. An orthogonal function $\mathrm{f}\left(\mathrm{x}_{\mathrm{i}}, \mathrm{y}_{\mathrm{i}}\right)$ is determined according the existence of obstacles in the fixed area map. The path is determined by solving the following nonlinear optimization problem:

$\min c=\sum_{i=1}^{n} d_{i}$

subjected to

$f\left(x_{i}, y_{i}\right)= \begin{cases}0 & \text { free cells (no obstacle) } \\ 1 & \text { occupied cells (obstacle) }\end{cases}$

Where

$d_{i}=\sqrt{\left(X_{i}-X_{i+1}\right)^{2}+\left(Y_{i}-Y_{i+1}\right)^{2}}$

$c$ is the sum of consecutive distance from start to the target, $n$ is the number of points in the path, $\left(x_{1}, y_{1}\right)$ is the intial location of the blind people and $\left(x_{2}, y_{2}\right)$ is the next node in the path subjected to $f\left(x_{i}, y_{i}\right) \neq 1$.

Accordingly, the path planning issue represent an optimization problem for obtaining the optimal cost value under limited conditions in mathematics necessarily, and it is modeled as a nonlinear optimal problem in numerical methods.

\subsection{Proposed solution technique based on GA and $A^{*}$ as a fitness function}

A proposed path planning algorithm is clarified. The algorithm used genetic search as well as $A^{*}$ algorithm as fitness function. The shortcoming of the $A^{*}$ algorithm is that it supposes a high dimensional set of solutions (paths) that can be generated. Thus, the computational time and complexity of the algorithm is high. The genetic search can overcome this problem by only selecting some paths that can be searched to determine the optimum path. Thus, the evaluation function $\mathrm{f}(\mathrm{n})$ of the $A^{*}$ algorithm is used as the fitness function of the genetic algorithm the proposed system steps are shown in Algorithm 1. 


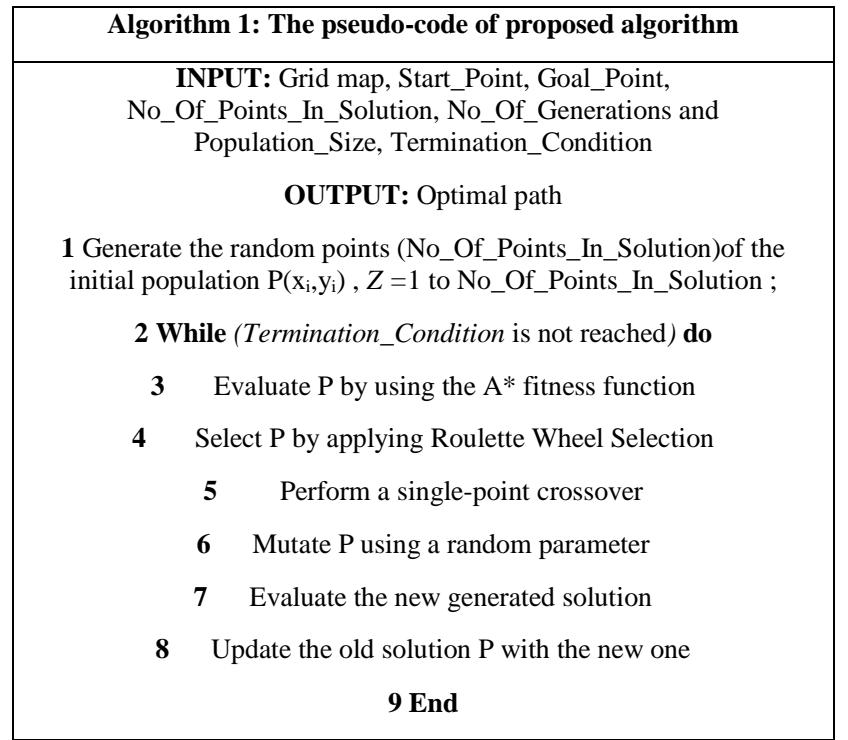

After creating the initial population randomly [57], the GA must decide the performance of every individual by employing an adaptive function that assigns to every possible solution a given fitness value which determines its quality. This fitness function has to consider many criteria, like distance, smoothness, safety, etc. Defining a suitable fitness function represents a crucial task as the GA employs the information produced by such function to select individuals for the reproduction process, mutation, and choose the best solution in the final population based on its fitness value at the GA process ending [58]. The proposed fitness function is demonstrated in Algorithm 2.

\begin{tabular}{|c|}
\hline Algorithm 2: Fitness function Pseudo-code \\
\hline Inputs: The initial start $S$ \\
\hline The end goal G \\
\hline The grid $\mathrm{D}$, \\
\hline The points in solution $\mathrm{k}$ \\
\hline Outputs: \\
\hline Optimal path, \\
\hline Cost, \\
\hline$\%$ Initialization step \\
\hline $\begin{array}{l}\text { Generate an initial population } \mathrm{O} \text { of random points } \mathrm{O}\left(\mathrm{x}_{\mathrm{i}}, \mathrm{y}_{\mathrm{i}}\right) \text { and } \\
\qquad(\mathrm{S}, \mathrm{G}) \text { points }\end{array}$ \\
\hline For $\mathrm{i}=1$ to $\mathrm{k}+2$ \\
\hline $\begin{array}{l}\text { \%Connect the points which have certain distance (connections) to } \\
\text { each other }\end{array}$ \\
\hline IF Is Feasible $\left(\mathrm{O}_{\mathrm{i}}, \mathrm{O}_{\mathrm{i}+1}\right)$ Then add edge \\
\hline End \\
\hline$\%$ Set of the nodes evaluated already \\
\hline$\%$ Set of nodes that will be evaluated \\
\hline Navigate_form $=0 \%$ the navigated nodes map \\
\hline Break $=1+1 /$ length $(D)+$ width $(D)$ \\
\hline$\%$ The breaking ties coefficients \\
\hline$B_{-}$score $[S]=0 \%$ Cost from the starting point along optimal \\
\hline
\end{tabular}

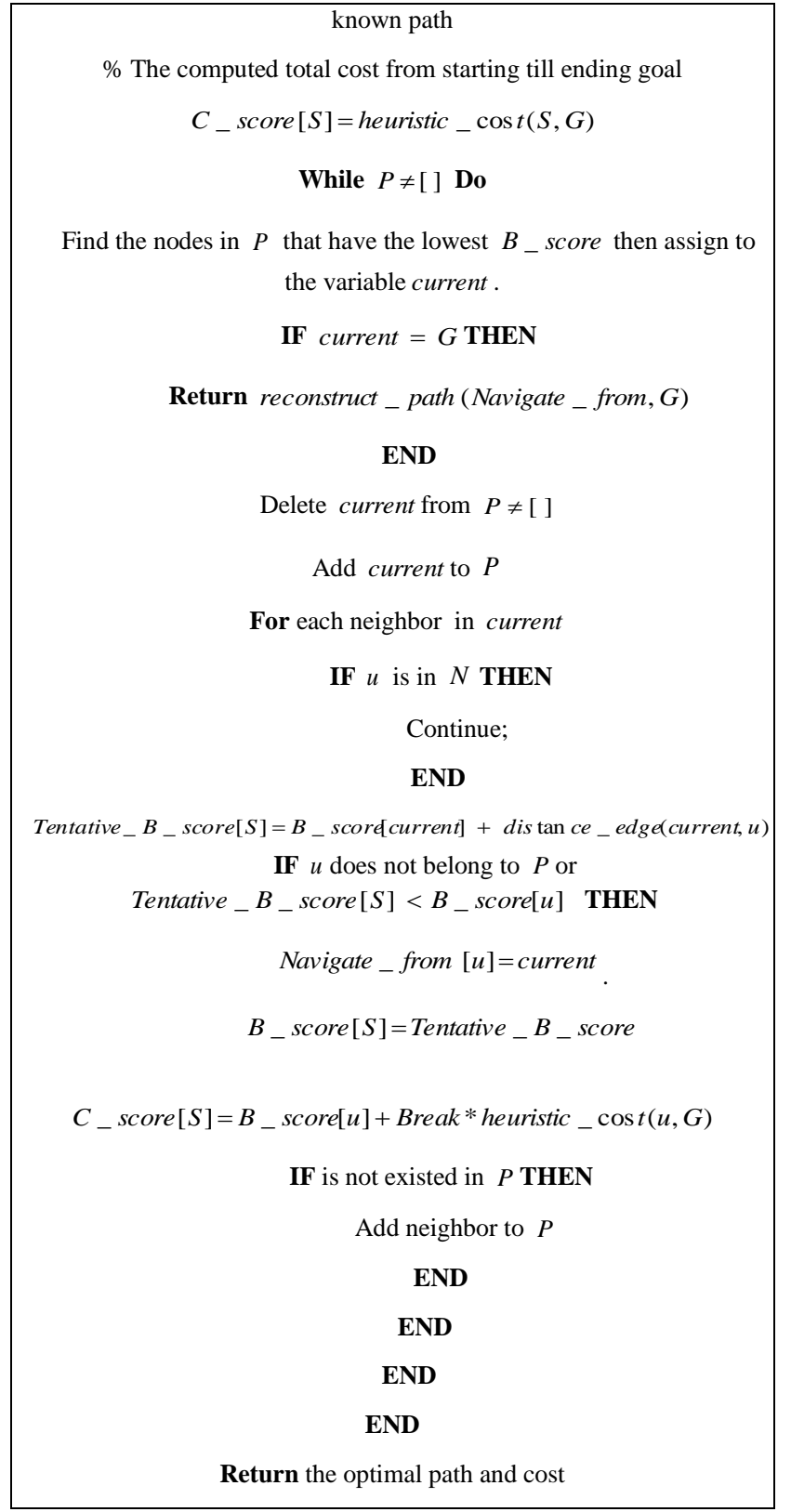

In this step, the evaluation function $F$ of the $A^{*}$ algorithm is used as a fitness function for the genetic algorithm.

Figure 5 demonstrates the application of GA and the evolution GA algorithm for defining the correct path. From the figure, the fitness function of the evolution algorithm selects the optimal points leading to the shortest path and ignores the other points that may increase the distance from start to end points and reduce the number of turns within the path. On the contrary, the GA fitness function selects only the points leading to the correct path regardless of the distance between starting and ending points. Therefore, the GA increases the number of turns in the path as shown in right of Figure 5.

In selection process, the selection is based upon roulette wheel selection [59] where every individual is associated with a portion proportional to its fitness function.

For next generations, the single-point crossover is used. The next process is mutation, is applied for enhancing the diversity and preventing algorithm premature convergence. Generally, this operation randomly chooses a position (gene) 
to replace it with a different new gene which is not existed in the path.

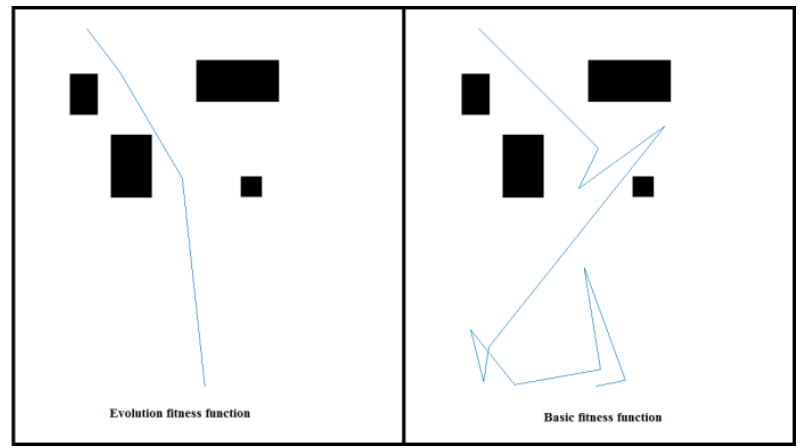

Figure 5 Evolution fitness function and basic fitness function for GA

Finally, according to path planning results the proposed system provides a voice feedback about the safe path that the blind person can walk through.

\subsection{Path guidance}

The final task for the proposed system is the guidance. It simply includes translating the resulting path into voice instructions comprising a series of directions and distances which represent a feedback to the blind person to direct his/her movements to the required destination.

After path planning algorithm is applied, the optimal path is generated for the blind person through selecting the path from some nodes which represent the best possible route for the blind person to walk through. It consists of two turning points from start point to target point as shown in Figure 6.

In this context, the blind person needs to start from starting point $\mathrm{A}$ to reach the target point $\mathrm{T}$. This requires finding the optimal path from A to T. Blind people should walk step by step till he/she reaches the target. However he/she may deviate from the critical path. This can be avoided through step by step critical path by utilizing voice guidance, if required, till he/she reaches the target point. This step can be explained as shown in Figure 7.

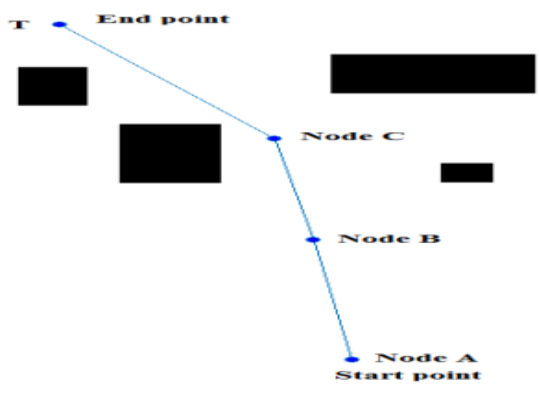

Figure 6 path planning turning points

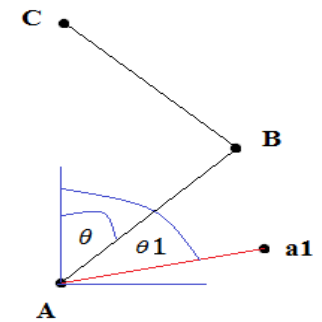

Figure 7 Way point guidance

Assume that blind people's posture is $(x, y, \theta)$, the $x, y$ is the position coordinates of the any point i.e. $\mathrm{A}, \mathrm{B}$, or $\mathrm{C}$, and $\theta$ is the direction angle of the blind person path and is determined using the following equation[60]:

$\theta=\tan ^{-1}\left(\frac{y_{i}-y}{x_{i}-x}\right)$

Where $W P\left(x_{i}, y_{i}\right)$, for $(i=1 \ldots \ldots N)$ is the given set of way points which is then translated to voice feedback for the blind people. The route is initially proposed supposing no obstacle from the path till the destination, because the person is unaware of the obstacles. After identifying route, the path identified is thereafter traversed by searching for obstacles. If any obstacle is found in the path, a new route is computed from the person's current position (supposing the current position is the beginning node) till the destination, so as to reroute the blind person.

\section{EXPERIMENTAL RESULTS}

In terms of computational time, this paper compared the performance of the proposed path planning algorithm to the conventional GA, as well as the $\mathrm{A}^{*}$ algorithm. The results demonstrated in Table 2. From the table, it is obvious that $\mathrm{A}^{*}$ algorithm outperforms the two compared algorithms respecting the shortest path. However, it requires a long computational time to get the shortest distance. On the other hand, the GA time is impressive in comparison to $\mathrm{A}^{*}$. Besides that, the distance from starting to target points does not differ much in comparison to $\mathrm{A}^{*}$. On the contrary, the proposed path planning algorithm outperforms the two other algorithms in terms of a computational time and a path length. Figure 8 shows the optimal path taken from the three comparable methods.

Table 2. The comparable results of the proposed path planning algorithm, GA, and $\mathrm{A} *$ algorithm

\begin{tabular}{|c|c|c|}
\hline Methodology & Computational time & Path Length \\
\hline GA & $75 \mathrm{~s}$ & $682 \mathrm{~cm}$ \\
\hline A* $^{*}$ & $191 \mathrm{~s}$ & $578 \mathrm{~cm}$ \\
\hline Proposed algorithm & $3 \mathrm{~s}$ & $561 \mathrm{~cm}$ \\
\hline
\end{tabular}




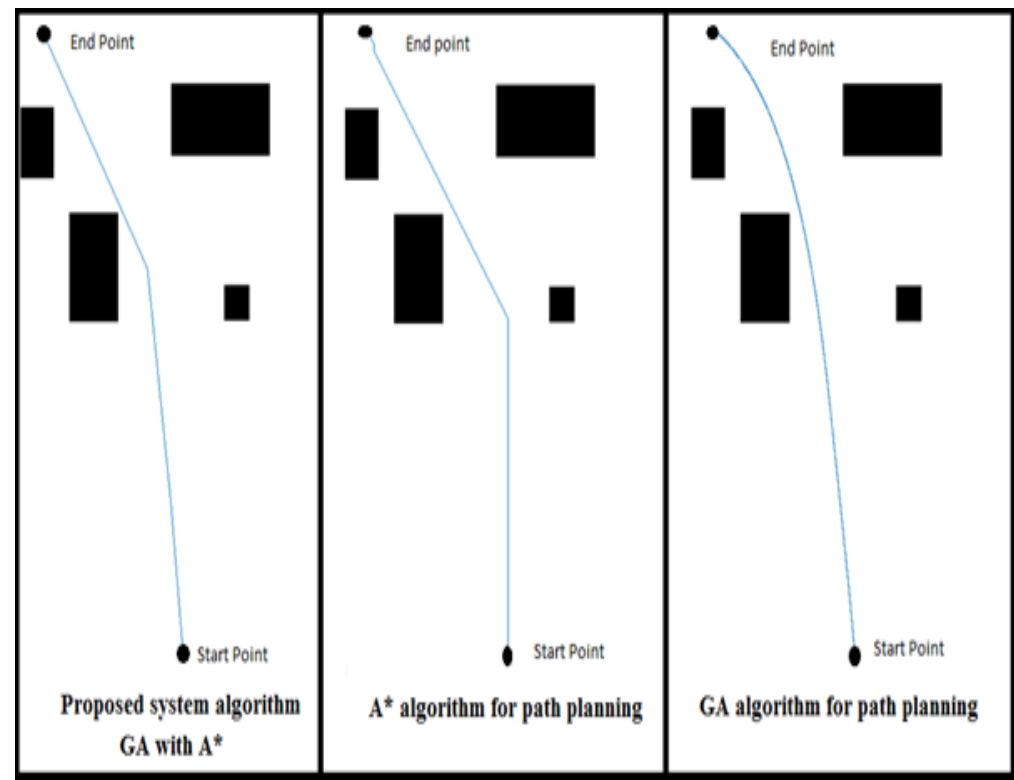

Figure 8 path planning comparisons of proposed algorithm, A* algorithm, and GA algorithm for path planning.

\section{CONCLUSIONS}

This paper proposes a novel system for the guidance of blind people through indoor environment based on a proposed path planning algorithm. The path planning system receives the grid map of the indoor place and calculates a safe path for the user to walk through. The path planning algorithm proposed in this paper is GA including $\mathrm{A}^{*}$ as fitness function to get the optimal path. The performance of the proposed path planning algorithm was compared to the GA, as well as the $A^{*}$ algorithm. Experimental results demonstrated that the proposed path planning algorithm outperforms the two other algorithms in reference to computational time and the path length.

\section{REFERENCES}

[1] K. Hasan, Abdullah-Al-Nahid and K. Reza, "Path planning algorithm development for autonomous vacuum cleaner robots", 2014 International Conference on Informatics, Electronics \& Vision (ICIEV), pp. 1-6, 2014.

[2] S. Mahmoudzadeh, D. Powers, K. Sammut, A. Lammas and A. Yazdani, "Optimal route planning with prioritized task scheduling for AUV missions", 2015 IEEE International Symposium on Robotics and Intelligent Sensors (IRIS), 2015.

[3] R. Galvez, E. Dadios and A. Bandala, "Path planning for quadrotor UAV using genetic algorithm", 2014 International Conference on Humanoid, Nanotechnology, Information Technology, Communication and Control, Environment and Management (HNICEM), 2014.

[4] Y. Lee and G. Medioni, "Wearable RGBD Indoor Navigation System for the Blind", Computer Vision ECCV 2014 Workshops, pp. 493-508, 2015.

[5] U. Orozco-Rosas, O. Montiel and R. Sepúlveda, "Mobile robot path planning using membrane evolutionary artificial potential field", Applied Soft Computing, vol. 77, pp. 236-251, 2019.

[6] J. Han and Y. Seo, "Mobile robot path planning with surrounding point set and path improvement", Applied Soft Computing, vol. 57, pp. 35-47, 2017.
[7] Y. Wang, D. Mulvaney, I. Sillitoe and E. Swere, "Robot Navigation by Waypoints", Journal of Intelligent and Robotic Systems, vol. 52, no. 2, pp. 175-207, 2008.

[8] "Vision impairment and blindness", Who.int, 2019. [Online]. Available: https://www.who.int/newsroom/fact-sheets/detail/blindness-and-visual-impairment. [Accessed: 09- Mar- 2019].

[9] D. Ni, A. Song, L. Tian, X. Xu and D. Chen, "A Walking Assistant Robotic System for the Visually Impaired Based on Computer Vision and Tactile Perception", International Journal of Social Robotics, vol. 7, no. 5, pp. 617-628, 2015.

[10] M. Yakoubi and M. Laskri, "The path planning of cleaner robot for coverage region using Genetic Algorithms", Journal of Innovation in Digital Ecosystems, vol. 3, no. 1, pp. 37-43, 2016.

[11] G. Kumar, U. Painumgal, M. Kumar and K. Rajesh, "Autonomous Underwater Vehicle for Vision Based Tracking", Procedia Computer Science, vol. 133, pp. 169-180, 2018.

[12] J. Tao, C. Zhong, L. Gao and H. Deng, "A Study on Path Planning of Unmanned Aerial Vehicle Based on Improved Genetic Algorithm", 2016 8th International Conference on Intelligent Human-Machine Systems and Cybernetics (IHMSC), 2016.

[13] M. Zafar and J. Mohanta, "Methodology for Path Planning and Optimization of Mobile Robots: A Review", Procedia Computer Science, vol. 133, pp. 141$152,2018$.

[14] F. Matoui, B. Boussaid, B. Metoui, G. Frej and M. Abdelkrim, "Path planning of a group of robots with potential field approach: decentralized architecture", IFAC-PapersOnLine, vol. 50, no. 1, pp. 11473-11478, 2017.

[15] Z. Wang and J. Cai, "Probabilistic roadmap method for path-planning in radioactive environment of nuclear facilities", Progress in Nuclear Energy, vol. 109, pp. 113120, 2018. 
[16] A. Panov, K. Yakovlev and R. Suvorov, "Grid Path Planning with Deep Reinforcement Learning: Preliminary Results", Procedia Computer Science, vol. 123, pp. 347-353, 2018.

[17] W. Wang, H. Deng and X. Wu, "Path planning of loaded pin-jointed bar mechanisms using Rapidly-exploring Random Tree method", Computers \& Structures, vol. 209, pp. 65-73, 2018.

[18] S. Mohammad, M. Jeffril and N. Sariff, "Mobile robot obstacle avoidance by using Fuzzy Logic technique", 2013 IEEE 3rd International Conference on System Engineering and Technology, 2013.

[19] H. Li, S. Yang and M. Seto, "Neural-Network-Based Path Planning for a Multirobot System With Moving Obstacles", IEEE Transactions on Systems, Man, and Cybernetics, Part C (Applications and Reviews), vol. 39, no. 4, pp. 410-419, 2009.

[20] X. Chen, Y. Kong, X. Fang and Q. Wu, "A fast two-stage ACO algorithm for robotic path planning", Neural Computing and Applications, vol. 22, no. 2, pp. 313-319, 2011.

[21] B. Patle, D. Parhi, A. Jagadeesh and S. Kashyap, "Matrix-Binary Codes based Genetic Algorithm for path planning of mobile robot", Computers \& Electrical Engineering, vol. 67, pp. 708-728, 2018.

[22] Y. Zhang, D. Gong and J. Zhang, "Robot path planning in uncertain environment using multi-objective particle swarm optimization", Neurocomputing, vol. 103, pp. 172-185, 2013

[23] N. Fallah, I. Apostolopoulos, K. Bekris and E. Folmer, "Indoor Human Navigation Systems: A Survey", Interacting with Computers, vol. 25, no. 1, pp. $21-33,2013$.

[24] M. Hosny, R. Alsarrani and A. Najjar, "Indoor Wheelchair Navigation for the Visually Impaired", Communications in Computer and Information Science, pp. 411-417, 2015.

[25] J. Bai, S. Lian, Z. Liu, K. Wang and D. Liu, "VirtualBlind-Road Following-Based Wearable Navigation Device for Blind People", IEEE Transactions on Consumer Electronics, vol. 64, no. 1, pp. 136-143, 2018.

[26] T. Gao Smith, C. Rose, J. Nolen, D. Pierce and A. Sherman, "Interactive Navigation System for the Visually Impaired with Auditory and Haptic Cues in Crosswalks, Indoors and Urban Areas", Communications in Computer and Information Science, pp. 539-545, 2015.

[27] D. Nandini and K. Seeja, "A novel path planning algorithm for visually impaired people", Journal of King Saud University - Computer and Information Sciences, 2017.

[28] M. Eichhorn, "Optimal routing strategies for autonomous underwater vehicles in time-varying environment", Robotics and Autonomous Systems, vol. 67, pp. 33-43, 2015.

[29] S. Choi, S. Lee, H. Viet and T. Chung, "B-Theta*: an Efficient Online Coverage Algorithm for Autonomous Cleaning Robots", Journal of Intelligent \& Robotic Systems, vol. 87, no. 2, pp. 265-290, 2017.
[30] M. Yakoubi and M. Laskri, "The path planning of cleaner robot for coverage region using Genetic Algorithms", Journal of Innovation in Digital Ecosystems, vol. 3, no. 1, pp. 37-43, 2016.

[31] M. Alajlan, A. Koubaa, I. Chaari, H. Bennaceur and A. Ammar, "Global path planning for mobile robots in large-scale grid environments using genetic algorithms", 2013 International Conference on Individual and Collective Behaviors in Robotics (ICBR), 2013.

[32] Pu Shi and Yujie Cui, "Dynamic path planning for mobile robot based on genetic algorithm in unknown environment", 2010 Chinese Control and Decision Conference, 2010.

[33] T. Yusof, S. Toha and H. Yusof, "Path Planning for Visually Impaired People in an Unfamiliar Environment Using Particle Swarm Optimization", Procedia Computer Science, vol. 76, pp. 80-86, 2015.

[34] Y. Zhang, D. Gong and J. Zhang, "Robot path planning in uncertain environment using multi-objective particle swarm optimization", Neurocomputing, vol. 103, pp. 172-185, 2013

[35] S. Mahmoudzadeh, D. Powers, K. Sammut, A. Lammas and A. Yazdani, "Optimal route planning with prioritized task scheduling for AUV missions", 2015 IEEE International Symposium on Robotics and Intelligent Sensors (IRIS), 2015.

[36] Y. Zhuang, S. Sharma, B. Subudhi, H. Huang and J. Wan, "Efficient collision-free path planning for autonomous underwater vehicles in dynamic environments with a hybrid optimization algorithm", Ocean Engineering, vol. 127, pp. 190-199, 2016.

[37] S. Primatesta, L. Cuomo, G. Guglieri and A. Rizzo, "An Innovative Algorithm to Estimate Risk Optimum Path for Unmanned Aerial Vehicles in Urban Environments", Transportation Research Procedia, vol 35, pp. 44-53, 2018.

[38] L. Yu, Z. Wei, Z. Wang, Y. Hu and H. Wang, "Path optimization of AUV based on smooth-RRT algorithm", 2017 IEEE International Conference on Mechatronics and Automation (ICMA), 2017.

[39] P. Yao and H. Wang, "Dynamic Adaptive Ant Lion Optimizer applied to route planning for unmanned aerial vehicle", Soft Computing, vol. 21, no. 18, pp. 54755488,2016

[40] J. Velagic, D. Delimustafic and D. Osmankovic, "Mobile robot navigation system based on Probabilistic Road Map (PRM) with Halton sampling of configuration space", 2014 IEEE 23rd International Symposium on Industrial Electronics (ISIE), 2014.

[41] E. Yanmaz, R. Kuschnig, M. Quaritsch, C. Bettstetter and B. Rinner, "On path planning strategies for networked unmanned aerial vehicles", 2011 IEEE Conference on Computer Communications Workshops (INFOCOM WKSHPS), 2011.

[42] R. M., Ghoniem. "Deep Genetic Algorithm-based Voice Pathology Diagnostic System", Natural Language Processing and Information Systems Lecture Notes in Computer Science. (2019). 
[43] A. Jayaprakash and C. Keziselvavijila. 2019. Feature Selection using Ant Colony Optimization (ACO) and Road Sign Detection and Recognition (RSDR) System. Cognitive Systems Research(2019). DOI:http://dx.doi.org/10.1016/j.cogsys.2019.04.002.

[44] R. M., Ghoniem \& K. Shaalan, (2017). "FCSR - Fuzzy Continuous Speech Recognition Approach for Identifying Laryngeal Pathologies Using New Weighted Spectrum Features". Proceedings of the International Conference on Advanced Intelligent Systems and Informatics 2017 Advances in Intelligent Systems and Computing, 384-395. http://doi.org/10.1007/978-3-31964861-3_36.

[45] T. Mac, C. Copot, D. Tran and R. De Keyser, "Heuristic approaches in robot path planning: A survey", Robotics and Autonomous Systems, vol. 86, pp. 13-28, 2016.

[46] C. Lamini, S. Benhlima and A. Elbekri, "Genetic Algorithm Based Approach for Autonomous Mobile Robot Path Planning", Procedia Computer Science, vol. 127, pp. 180-189, 2018.

[47] J. Yao, C. Lin, X. Xie, A. Wang and C. Hung, "Path Planning for Virtual Human Motion Using Improved A* Star Algorithm", 2010 Seventh International Conference on Information Technology: New Generations, 2010.

[48] F. Duchoň et al., "Path Planning with Modified a Star Algorithm for a Mobile Robot", Procedia Engineering, vol. 96, pp. 59-69, 2014.

[49] I. Wojnicki, S. Ernst and W. Turek, "A Robust Heuristic for the Multidimensional A-star/Wavefront Hybrid Planning Algorithm", Artificial Intelligence and Soft Computing, pp. 282-291, 2015.

[50] K. Da, L. Xiaoyu and Z. Bi, "Variable-step-length A* algorithm for path planning of mobile robot", 2017 29th Chinese Control And Decision Conference (CCDC), 2017.

[51] G. Klančar, A. Zdešar, S. Blažič and I. Škrjanc, "Path Planning", Wheeled Mobile Robotics, pp. 161-206, 2017.

[52] A. Tuncer and M. Yildirim, "Dynamic path planning of mobile robots with improved genetic algorithm", Computers \& Electrical Engineering, vol. 38, no. 6, pp. 1564-1572, 2012.

[53] M. Alajlan, A. Koubaa, I. Chaari, H. Bennaceur and A. Ammar, "Global path planning for mobile robots in large-scale grid environments using genetic algorithms", 2013 International Conference on Individual and Collective Behaviors in Robotics (ICBR), 2013.

[54] Shenlu Jiang, Zhonghua Hong, Yun Zhang, Yanling Han, Ruyan Zhou and Bo Shen, "Automatic path planning and navigation with stereo cameras", 2014 Third International Workshop on Earth Observation and Remote Sensing Applications (EORSA), 2014.

[55] I. Châari et al., "On the Adequacy of Tabu Search for Global Robot Path Planning Problem in Grid Environments", Procedia Computer Science, vol. 32, pp. 604-613, 2014.

[56] N. Eraghi, F. Lopez-Colino, A. de Castro and J. Garrido, "Path length comparison in grid maps of planning algorithms: HCTNav and Dijkstra", Design of Circuits and Integrated Systems, 2014

[57] Y. Miao, A. Khamis, F. Karray and M. Kamel, "A NOVEL APPROACH TO PATH PLANNING FOR AUTONOMOUS MOBILE ROBOTS", Control and Intelligent Systems, vol. 39, no. 4, 2011.

[58] Haiming Shen, "A study of welding robot path planning application based on Genetic Ant Colony Hybrid Algorithm", 2016 IEEE Advanced Information Management, Communicates, Electronic and Automation Control Conference (IMCEC), 2016.

[59] M. Gao, G. Shi, W. Li, Y. Wang and D. Liu, "An Improved Genetic Algorithm for Island Route Planning", Procedia Engineering, vol. 174, pp. 433-441, 2017.

[60] H. Kim, D. Kim, H. Kim, J. Shin and H. Myung, "An extended any-angle path planning algorithm for maintaining formation of multi-agent jellyfish elimination robot system", International Journal of Control, Automation and Systems, vol. 14, no. 2, pp. 598-607, 2016. 